\title{
Efficacy of embryo transfer on day 2 versus day 3 according to maternal age in patients with normal ovarian response
}

\author{
Jung-Woo Lee ${ }^{1,2}$, Jeong-Ho Cha', Sun-Hee Shin ${ }^{1,2}$, Yun-Jeong Kim', Seul-Ki Lee' ${ }^{1}$ Choon-keun Park' ${ }^{2}$ Kyung-Ah Pak', \\ Ji-Sung Yoon ', Seo-Young Park' \\ 'Agaon Fertility Clinic, Seoul; ${ }^{2}$ College of Animal Life Sciences, Kangwon National University, Chuncheon, Korea
}

Objective: Delaying embryo transfer (ET) enables us to select among the embryos available for transfer and is associated with positive effects on implantation and pregnancy outcomes. However, the optimal day for ET of human cleavage-stage embryos remains controversial.

Methods: A retrospective study of 3,124 in vitro fertilization/intracytoplasmic sperm injection cycles (2,440 patients) was conducted. We compared the effects of day 2 and $3 \mathrm{ET}$ on rates of implantation and pregnancy outcomes between young maternal age (YMA; $<38$ years old, $n=2,295)$ and old maternal age (OMA; $\geq 38$ years old, $n=829$ ) patient groups.

Results: The YMA and OMA groups did not differ in terms of patient characteristics except for the proportion of unexplained factor infertility, which was significantly greater in the OMA group, and the proportion of arrested embryos, which was significantly greater in the YMA group. However, the biochemical pregnancy, clinical pregnancy, ongoing pregnancy, abortion, and implantation rates per cycle were not significantly different between day 2 and 3 ET in the YMA group or the OMA group.

Conclusion: We suggest that offering patients the opportunity to decide which day would be suitable for ET could be part of a patient-friendly protocol that takes into consideration an infertile woman's circumstances and work schedule by allowing ET to be performed on day 2 instead of the traditional transfer on day 3.

Keywords: Clinical pregnancy; Embryo transfer; Implantation; Maternal age

\section{Introduction}

Embryo transfer (ET) of human cleavage-stage embryos is commonly conducted on the third day after oocyte retrieval. One of the main reasons why ET occurs when human embryos are at the morula stage is that the fertilized oocyte is normally located in the endometrium on days 4 to 5 after ovulation [1]. Therefore, the physiology of the intrauterine environment is more suitable for the developing morula on day 3 than it is on day 2 [2]. In general, delaying ET may

Received: May 30, 2017 · Revised: Jul 5, 2017 · Accepted: Aug 4, 2017 Corresponding author: Jeong-Ho Cha

Agaon Fertility Clinic, 8F, 79 Digital-ro 32-gil, Guro-gu, Seoul 08391, Korea Tel: +82-2-864-7500 Fax:+82-2-864-7550 E-mail: ivfcha@naver.com

This is an Open Access article distributed under the terms of the Creative Commons Attribution Non-Commercial License (http://creativecommons.org/licenses/by-nc/4.0/) which permits unrestricted non-commercial use, distribution, and reproduction in any medium, provided the original work is properly cited. therefore be appropriate if embryo development is satisfactory during in vitro culture. Delaying ET can increase the possibility of successful implantation and also make it possible to select the most viable embryos for transfer [3]. Previous studies found that day 3 ET led to higher rates of pregnancy and implantation than day $2 \mathrm{ET}$ [3-5].

However, delaying ET and leaving embryos in in vitro culture conditions could have negative effects on embryo development and could lead to a decreased number of viable embryos available for transfer [2]. Shen et al. [6] reported that day $2 \mathrm{ET}$ in patients $<40$ years of age was associated with a significant increase in the ongoing pregnancy rate, with a concomitant decrease in the miscarriage rate. Moreover, a study showed that pregnancy outcomes in poor responders were improved by day 2 ET [7]. However, the optimal day for ET of human cleavage-stage embryos remains controversial. Some recent studies found no difference in the implantation and pregnancy rates be- 
tween day 2 and 3 ET $[1,8,9]$.

Therefore, we compared the influence of day 2 and 3 ET on the rates of implantation and pregnancy outcomes in young maternal age (YMA) and old maternal age (OMA) patient groups.

\section{Methods}

\section{Participants}

A retrospective study of 3,124 in vitro fertilization (IVF)/intracytoplasmic sperm injection (ICSI) cycles in patients with normal ovarian response ( $n=2,440$ patients) was conducted from January 2013 to December 2016 at Agaon Fertility Clinic, Seoul, Korea. Cycles were divided into groups according to maternal age and the day of ET (YMA: $<38$ years old; day $2 \mathrm{ET}, \mathrm{n}=271$ vs. day $3 \mathrm{ET}, \mathrm{n}=2,024$ and OMA: $\geq 38$ years old; day $2 E T, n=106$ vs. day $3 E T, n=723$ ) (Figure 1 ). The criteria for performing ET on day 2 were as follows: (1) if day 3 ET would have fallen on a holiday, or (2) if the patient could not undergo ET on day 3. Cycles with a genetic diagnosis, frozen-thawed embryos, oocyte donation, oocyte activation, repeated implantation failure ( $\geq 3$ times), and that used surrogate mothers were excluded from our data. Institutional Review Board approval of Agaon Fertility Clinic was obtained for this study (IRB No. Agaon IRB-17-001).

\section{Ovarian stimulation and IVF/ICSI procedures}

All women were treated with either a gonadotropin-releasing hormone (GnRH) agonist long protocol or a GnRH antagonist (Cetrotide, Merck Serono, Roma, Italy) protocol. Human chorionic gonadotropin (hCG; Ovidrel, Merck Serono) was given to the patients 36 hours prior to oocyte retrieval. Oocytes were retrieved via transvaginal ultrasound guidance with a 19-gauge needle (Dukwoo Medical, Hwaseong, Korea). Mature oocytes were inseminated with either ICSI or conventional insemination according to the state of the spermatozoa and oocytes, as well as the patient's previous IVF history [10]. The pres-

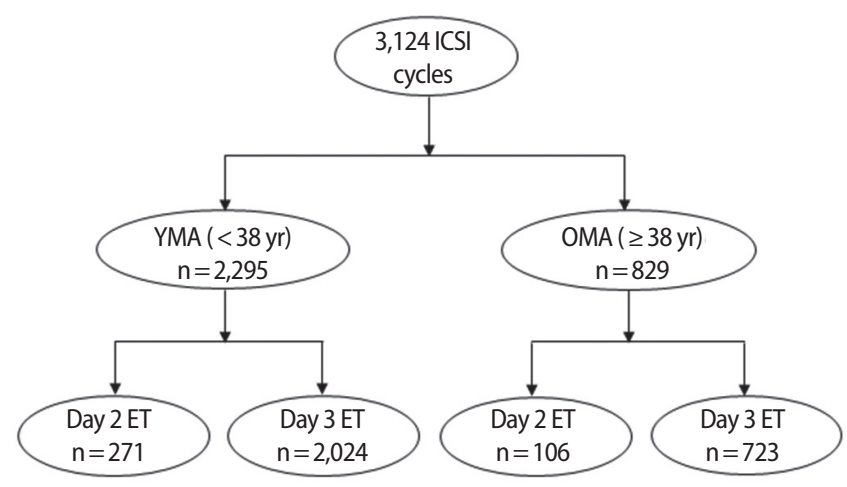

Figure 1. Flow diagram of the study design. ICSI, intracytoplasmic sperm injection; YMA, young maternal age; OMA, old maternal age; ET, embryo transfer. ence of two pronuclei was observed at 17 to 19 hours after insemination. Normal fertilized zygotes were cultured in 30- $\mu \mathrm{L}$ micro-drops in either a sequential medium (Vitrolife, Gothenburg, Sweden) or a 1-step medium (Life Global, Belgium) and overlaid with paraffin oil (Vitrolife) in an atmosphere of $6 \% \mathrm{CO}_{2}, 5 \% \mathrm{O}_{2}$, and $95 \%$ humidity at $37^{\circ} \mathrm{C}$.

\section{ET and clinical outcomes}

Either two or three embryos, which are the number of embryos recommended by the Ministry of Health and Welfare in Korea, were transferred either on day 2 or day 3 to the uterus. The available embryos were evaluated for use in ET based on the viable blastomere number, fragmentation ratio, and symmetrical pattern of blastomeres, and ET was performed using a catheter (COOK Medical, Brisbane, Australia). The women's endometria were prepared according to artificial cycle protocols with exogenous estrogens. Progesterone in oil (50 mg/day) was administered on a daily basis until the endometrial thickness reached $\geq 8 \mathrm{~mm}$. A positive pregnancy was defined as the presence of $\beta$-hCG ( $\geq 10 \mathrm{mlU} / \mathrm{mL}$ ) in the serum 14 days after oocyte retrieval. Successful implantation and positive clinical pregnancy were determined when ultrasonography showed the presence of a gestational sac at 6 weeks of pregnancy. Abortion was defined as fetal loss before 10 weeks of gestation. Ongoing pregnancy was defined as a positive pregnancy test followed by the presence of a heart-beat on an ultrasound examination at 10 weeks of gestation.

\section{Statistical analysis}

Data were analyzed using SPSS ver. 23.0 (IBM Corp., Armonk, NY, USA). The statistical differences between groups were assessed with the independent samples $t$-test for parametric data. The chi-square test was used to compare differences in rates. The $p$-values $<0.05$ were defined as representing a significant difference.

\section{Results}

A total of 3,124 cycles were included in the study. The maternal age classifications (i.e., YMA vs. OMA) were made according to the classification criteria proposed by Stoop et al. [11]. The YMA and OMA groups did not differ significantly in terms of the female partner's age, the male partner's age, the number of previous IVF attempts, or type of infertility, except that unexplained factor infertility was significantly more common in the OMA group. Nevertheless, the numbers of retrieved, matured, and fertilized oocytes and embryos transferred were similar in both groups (Table 1).

As shown in Table 1, the proportion of arrested embryos was significantly higher for day 3 ET than for day 2 ET in the YMA group (4.2\% vs. $3.0 \%, p=0.015$ ) and it was also slightly higher in the OMA group 
Table 1. Characteristics of patients who underwent day 2 versus day 3 ET

\begin{tabular}{|c|c|c|c|c|c|c|}
\hline \multirow{2}{*}{ Variable } & \multicolumn{3}{|c|}{ Maternal age $<38 \mathrm{yr}$} & \multicolumn{3}{|c|}{ Maternal age $\geq 38 \mathrm{yr}$} \\
\hline & Day $2 \mathrm{ET}$ & Day 3 ET & $p$-value & Day $2 \mathrm{ET}$ & Day 3 ET & $p$-value \\
\hline No. of cycles & 271 & 2,024 & - & 106 & 723 & - \\
\hline Female age (yr) & $33.1 \pm 2.5$ & $33.3 \pm 2.5$ & 0.123 & $40.0 \pm 2.0$ & $40.3 \pm 2.0$ & 0.201 \\
\hline Male age (yr) & $35.3 \pm 3.5$ & $35.4 \pm 3.4$ & 0.851 & $41.5 \pm 5.0$ & $40.9 \pm 4.0$ & 0.147 \\
\hline No. of IVF attempts & $0.8 \pm 1.0$ & $0.7 \pm 1.0$ & 0.471 & $1.1 \pm 1.0$ & $1.1 \pm 1.1$ & 0.469 \\
\hline \multicolumn{7}{|l|}{ Cause of infertility } \\
\hline Female factor & $98(36.2)$ & $787(38.9)$ & 0.387 & $72(67.9)$ & $437(60.4)$ & 0.139 \\
\hline Male factor & $41(15.1)$ & $343(16.9)$ & 0.452 & $3(2.8)$ & $21(2.9)$ & 0.966 \\
\hline Mixed factor & $29(10.7)$ & $168(8.3)$ & 0.185 & $16(15.1)$ & $84(11.6)$ & 0.305 \\
\hline Unexplained factor & $103(38.0)$ & $726(35.9)$ & 0.491 & $15(14.2)$ & $181(25.0)^{a)}$ & 0.014 \\
\hline No. of oocytes retrieved & $9.8 \pm 6.1$ & $10.2 \pm 6.1$ & 0.356 & $6.2 \pm 4.5$ & $6.6 \pm 4.6$ & 0.470 \\
\hline No. of oocytes matured & 2,411/2,676 (90.1) & $18,624 / 20,718(89.9)$ & 0.741 & $603 / 660(91.4)$ & $4,328 / 4,753(91.1)$ & 0.796 \\
\hline No. of oocytes fertilized & 1,831/2,411 (75.9) & $13,966 / 18,624(75.0)$ & 0.308 & $429 / 603(71.1)$ & $3,180 / 4,328(73.5)$ & 0.226 \\
\hline No. of embryos arrested on day $3^{\text {b) }}$ & $55 / 1,831(3.0)$ & $586 / 13,966(4.2)^{a)}$ & 0.015 & $12 / 429(2.8)$ & $151 / 3,180(4.7)$ & 0.068 \\
\hline No. of embryos transferred & $2.0 \pm 0.5$ & $2.0 \pm 0.5$ & 0.574 & $2.1 \pm 0.7$ & $2.0 \pm 0.6$ & 0.314 \\
\hline
\end{tabular}

Values are presented as mean \pm standard deviation or number (\%).

ET, embryo transfer; IVF, in vitro fertilization.

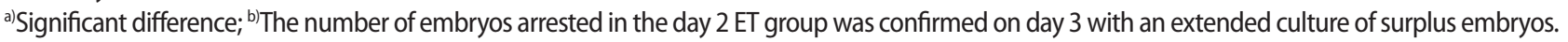

Table 2. Pregnancy outcomes of day 2 versus day $3 \mathrm{ET}$ according to maternal age

\begin{tabular}{|c|c|c|c|c|c|c|}
\hline \multirow{2}{*}{ Variable } & \multicolumn{3}{|c|}{ Maternal age $<38 \mathrm{yr}$} & \multicolumn{3}{|c|}{ Maternal age $\geq 38 \mathrm{yr}$} \\
\hline & Day $2 \mathrm{ET}$ & Day $3 \mathrm{ET}$ & $p$-value & Day $2 \mathrm{ET}$ & Day $3 \mathrm{ET}$ & $p$-value \\
\hline Biochemical pregnancy & $132(48.7)$ & $927(45.8)$ & 0.367 & $26(24.5)$ & $179(24.8)$ & 0.959 \\
\hline Clinical pregnancy & $111(41.0)$ & $782(38.6)$ & 0.461 & $22(20.8)$ & $132(18.3)$ & 0.537 \\
\hline Ongoing pregnancy & $100(36.9)$ & $691(34.1)$ & 0.369 & $16(15.1)$ & $111(15.4)$ & 0.945 \\
\hline Abortion & $11(9.9)$ & $91(11.6)$ & 0.592 & $6(27.3)$ & $21(15.9)$ & 0.194 \\
\hline Implantation & $142(26.0)$ & $1,012(24.6)$ & 0.477 & $24(10.9)$ & $163(11.2)$ & 0.882 \\
\hline
\end{tabular}

Values are presented as number (\%).

ET, embryo transfer.

( $4.7 \%$ vs. $2.8 \%, p=0.068)$. However, there was no statistically significant difference in the OMA group. In addition, the biochemical pregnancy, clinical pregnancy, ongoing pregnancy, abortion, and implantation rates per cycle were not significantly different between day 2 and 3 ET in the YMA group or the OMA group (Table 2).

\section{Discussion}

In this study, we compared the rates of implantation and clinical outcomes between day 2 and $3 \mathrm{ET}$ in 3,124 IVF/ICSI cycles in order to determine which day of ET (i.e., day 2 vs. day 3 ) was preferable according to maternal age.

Several previous studies have been conducted to compare day 2 and $3 \mathrm{ET}$, but their results are contradictory $[1,8]$. A retrospective study reported that the pregnancy rate was not significantly different between day 2 and $3 \mathrm{ET}$, but the implantation rate was positively associated with day 3 ET in contrast to day 2 ET [4]. Some studies have reported that the rates of implantation and pregnancy for day 3 ET were higher than those for day $2 \mathrm{ET}$. Two reasons for this finding may be that the intrauterine environment on day 3 is more similar to the environment at the physiological time of uterine entry than the environment on day 2 , and that the most viable embryos can be selected for transfer $[5,12,13]$. In addition, there is a higher probability of discriminating arrested or slow-growing embryos when ET is performed on day 3 than when it is performed on day 2 [14].

However, a prospective randomized clinical trial study found that exposing embryos derived from poor responders to in vitro conditions negatively affected the potential for embryo development. Therefore, long-term in vitro culture may not be a valid method for all patients, especially patients who have few available oocytes and embryos [2]. Furthermore, an earlier study reported that the proportion of human embryos that were arrested between the 5- and 8-cell stages $(82.8 \%, \mathrm{n}=73)$ was higher than that in embryos between the 2- and 4-cell stages $(59.5 \%, n=50)$ [15]. 
Frankfurter et al. [7] suggested that pregnancy rates were improved with day 2 ET, and Shen et al. [6] reported that the ongoing pregnancy rate with day 2 ET was higher than that with day $3 \mathrm{ET}$, which could be explained by the significantly lower rate of miscarriage observed in patients who underwent day 2 ET. In 1984, Edwards et al. [16] reported that the incidence of miscarriage after day 3 ET was higher than that for day 2 ET. A previous meta-analysis showed that the clinical pregnancy rate was higher with day $3 \mathrm{ET}$, but the higher miscarriage rates with day 3 ET negated the increased pregnancy rate and resulted in no difference in live births [3].

Additionally, in animal studies, it has been found that the post-fertilization culture environment resulted in detrimental effects on the developing embryos [17-19]. Some studies have also demonstrated a high incidence of embryo wastage under in vitro conditions by comparing early embryo development in both in vivo and in vitro conditions [20-22]. However, this present study has shown that, in a comparison of day 2 and day $3 \mathrm{ET}$, there were no differences in the rates of implantation and clinical outcomes between the YMA and OMA groups. We think that improvements in the culture medium and culture environment may reduce the detrimental effects of extended culture on embryo development under in vitro conditions. Furthermore, Dayal et al. [9] reported that a short period of in vitro culture conditions provided no obvious benefit for early cleavagestage ET.

Some studies have found no differences in the rates of implantation and pregnancy between day 2 and 3 ET [12,23-25], as well as no significant difference in outcomes based on the day of ET in poor responders $[9,26]$. A recent retrospective study found no difference in the rates of biochemical pregnancy and clinical pregnancy between day 2 and 3 ET according to single or double ET [1], and our study supports those results.

In the results of the present study, the proportion of arrested embryos on day 3 was higher in the day 3 ET group than in the day 2 ET group. One reason for this might be that the potentially arrested embryo was transferred on day 2 rather than on day 3. However, according to a previous study, poor-quality embryos could be rescued inside the uterine environment [27]. Additionally, embryo arrest could be caused by a prolonged period in in vitro culture [15].

In the present study, the number of day 2 ET cycles was much smaller than the number of day 3 ET cycles. However, the sample size in our study was larger than that used in previous retrospective studies. We used the age of 38 years to define the YMA and OMA groups because oocyte quality is significantly lower after controlled ovarian stimulation starting at the age of 38 years, and it also decreases dramatically after the age of 40 years [11].

The proportion of infertile couples has been increasing annually. One of the main reasons for this is that more women are getting married later in life and more working women are putting off having children in Korea. In fact, a professional woman who desires to have children through infertility treatment may encounter difficulties when visiting an IVF clinic at an appointed date for ET because of her working environment and schedule. Therefore, the IVF clinic may reduce the psychological burden for everyone involved by allowing the patient to decide on the ET date (i.e., day 2 or day 3).

Previous research into the preferable date of ET for optimal and stable cleavage has shown insufficient evidence for an improvement in implantation and pregnancy rates between day 2 and 3 ET. However, in a recent review, Brown et al. [28] reported that there was no evidence of differences in the rates of live births, ongoing pregnancy, clinical pregnancy, multiple pregnancy, miscarriage, or ectopic pregnancy between day 2 and 3 ET. Therefore, our data provide reliable evidence to support this proposal.

We conclude that there was no difference in outcomes between day 2 and 3 ET in the YMA and OMA groups. In addition, we suggest that offering patients the opportunity to decide which day would be suitable for ET could be part of a patient-friendly protocol that takes into consideration an infertile woman's circumstances and work schedule by allowing ET to be performed on day 2 instead of the traditional transfer on day 3. Further studies are required to confirm our findings with respect to the rates of live birth and miscarriage outcomes.

\section{Conflict of interest}

No potential conflict of interest relevant to this article was reported.

\section{Acknowledgments}

The authors express special thanks to the staff of the Agaon Fertility Clinic.

\section{References}

1. Bastu E, Celik C, Keskin G, Buyru F. Evaluation of embryo transfer time (day 2 vs day 3 ) after imposed single embryo transfer legislation: when to transfer? J Obstet Gynaecol 2013;33:387-90.

2. Bahceci M, Ulug U, Ciray HN, Akman MA, Erden HF. Efficiency of changing the embryo transfer time from day 3 to day 2 among women with poor ovarian response: a prospective randomized trial. Fertil Steril 2006;86:81-5.

3. Oatway C, Gunby J, Daya S. Day three versus day two embryo transfer following in vitro fertilization or intracytoplasmic sperm injection. Cochrane Database Syst Rev 2004;(2):CD004378.

4. Dawson KJ, Conaghan J, Ostera GR, Winston RM, Hardy K. Delay- 
ing transfer to the third day post-insemination, to select non-arrested embryos, increases development to the fetal heart stage. Hum Reprod 1995;10:177-82.

5. Carrillo AJ, Lane B, Pridman DD, Risch PP, Pool TB, Silverman IH, et al. Improved clinical outcomes for in vitro fertilization with delay of embryo transfer from 48 to 72 hours after oocyte retrieval: use of glucose- and phosphate-free media. Fertil Steril 1998; 69:329-34.

6. Shen S, Rosen MP, Dobson AT, Fujimoto VY, McCulloch CE, Cedars MI. Day 2 transfer improves pregnancy outcome in in vitro fertilization cycles with few available embryos. Fertil Steril 2006;86: 44-50.

7. Frankfurter D, Keefe DL, Trimarchi JB. Day 2 embryo transfer improves IVF-ET outcome in the poor responder. Fertil Steril 2003; 80 Suppl 3:61.

8. Ashrafi M, Kiani K, Mirzaagha E, Shabani F. The pregnancy outcomes of day 2 versus day 3 embryo transfer: a cross-sectional study. Int J Fertil Steril 2007;1:47-54.

9. Dayal MB, Frankfurter D, Athanasiadis I, Peak D, Dubey A, Gindoff PR. Day 2 embryo transfer (ET) and day 3 ET afford similar reproductive outcomes in the poor responder. Fertil Steril 2011;95: 1130-2.

10. Lee JW, Cha JH, Shin SH, Kim YJ, Lee SK, Cha HJ, et al. Long cut straw provides stable the rates of survival, pregnancy and live birth for vitrification of human blasotcysts. Dev Reprod 2016; 20:219-25.

11. Stoop D, Ermini B, Polyzos NP, Haentjens P, De Vos M, Verheyen G, et al. Reproductive potential of a metaphase II oocyte retrieved after ovarian stimulation: an analysis of 23354 ICSI cycles. Hum Reprod 2012;27:2030-5.

12. Laverge H, De Sutter P, Van der Elst J, Dhont M. A prospective, randomized study comparing day 2 and day 3 embryo transfer in human IVF. Hum Reprod 2001;16:476-80.

13. Baruffi RL, Mauri AL, Petersen C, Felipe V, Franco Junior JG. Day 2 vs. day 3 embryo transfer after intracytoplasmic sperm injection: a prospective, randomized study. J Reprod Med 2003;48:631-4.

14. Suzuki T, Shibahara H, Hirano Y, Ohno A, Takamizawa S, Suzuki M. Randomized study comparing day 2 versus day 3 elective transfer of two good-quality embryos. Reprod Med Biol 2004;3:99104.

15. Almeida PA, Bolton VN. Cytogenetic analysis of human preimplantation embryos following developmental arrest in vitro. Reprod Fertil Dev 1998;10:505-13.

16. Edwards RG, Fishel SB, Cohen J, Fehilly CB, Purdy JM, Slater JM, et al. Factors influencing the success of in vitro fertilization for alleviating human infertility. J In Vitro Fert Embryo Transf 1984;1:3-
23.

17. Lonergan P, Pedersen HG, Rizos D, Greve T, Thomsen PD, Fair T, et al. Effect of the post-fertilization culture environment on the incidence of chromosome aberrations in bovine blastocysts. Biol Reprod 2004;71:1096-100.

18. Lonergan P, Rizos D, Gutierrez-Adan A, Fair T, Boland MP. Oocyte and embryo quality: effect of origin, culture conditions and gene expression patterns. Reprod Domest Anim 2003;38:25967.

19. Lonergan P, Rizos D, Kanka J, Nemcova L, Mbaye AM, Kingston M, et al. Temporal sensitivity of bovine embryos to culture environment after fertilization and the implications for blastocyst quality. Reproduction 2003;126:337-46.

20. Knijn HM, Gjorret JO, Vos PL, Hendriksen PJ, van der Weijden BC, Maddox-Hyttel $\mathrm{P}$, et al. Consequences of in vivo development and subsequent culture on apoptosis, cell number, and blastocyst formation in bovine embryos. Biol Reprod 2003;69:1371-8.

21. Neuber E, Chan AW, Dominko T, Simerly C, Schatten G. Cell allocation and cell death in blastocysts from nonhuman primates generated during in vitro fertilization and intracytoplasmic sperm injection. Fertil Steril 2002;77:1083-5.

22. Rizos D, Fair T, Papadopoulos S, Boland MP, Lonergan P. Developmental, qualitative, and ultrastructural differences between ovine and bovine embryos produced in vivo or in vitro. Mol Reprod Dev 2002;62:320-7.

23. Ertzeid G, Dale PO, Tanbo T, Storeng R, Kjekshus E, Abyholm T. Clinical outcome of day 2 versus day 3 embryo transfer using serum-free culture media: a prospective randomized study. J Assist Reprod Genet 1999;16:529-34.

24. Aboulghar MM, Aboulghar MA, Mansour RT, Serour Gl, Amin YM, Abou-Setta AM. Pregnancy rate is not improved by delaying embryo transfer from days 2 to 3 . Eur J Obstet Gynecol Reprod Biol 2003;107:176-9.

25. de los Santos MJ, Mercader A, Galan A, Albert C, Romero JL, Pellicer A. Implantation rates after two, three, or five days of embryo culture. Placenta 2003;24 Suppl B:S13-9.

26. Shahine LK, Milki AA, Westphal LM, Baker VL, Behr B, Lathi RB. Day 2 versus day 3 embryo transfer in poor responders: a prospective randomized trial. Fertil Steril 2011;95:330-2.

27. Coskun S, Hollanders J, Al-Hassan S, Al-Sufyan H, Al-Mayman H, Jaroudi K. Day 5 versus day 3 embryo transfer: a controlled randomized trial. Hum Reprod 2000;15:1947-52.

28. Brown J, Daya S, Matson P. Day three versus day two embryo transfer following in vitro fertilization or intracytoplasmic sperm injection. Cochrane Database Syst Rev 2016;12:CD004378. 